\title{
Schwerpunkt: Multiple Identitäten in modernen Gesellschaften
}

Die Frage nach Zugehörigkeiten und Identitäten beschäftigt die Menschen bereits seit Anbeginn der Zeit. Egal ob es sich um die Familie handelt, die Sprachgemeinschaft, die Nation usw.: Schon immer war es für Menschen wichtig, zu einer Gruppe dazuzugehören oder sich von Anderen abzugrenzen. Nur selten wurden dabei die Selbstverortung des Individuums sowie die Homogenität der Gruppe durch exogene Veränderungen infrage gestellt.

Gestalteten sich Zugehörigkeit und Identität in früheren Zeiten also noch vergleichsweise übersichtlich, sieht man sich in dieser Hinsicht heute mit einer neuen Unübersichtlichkeit konfrontiert. In modernen, globalisierten Gesellschaften werden altbewährte Gewissheiten brüchig, überlagern sich Zugehörigkeiten, verlieren identitätsstiftende Faktoren ihre Bindungskraft. Patchwork-Familien sind heute keine Seltenheit mehr, viele Menschen wachsen mehrsprachig auf, und aufgrund von Migrationsbewegungen hat die (eine) Nation als Bezugspunkt an Bedeutung verloren. Doch nicht nur die Beziehung innerhalb oder zu anderen Gruppen ist Ausdruck von Identität. Identität bezieht sich zu allererst auf das Individuum selbst, auf jede einzelne Person. Kaum etwas drückt diesen Aspekt von Identität besser aus als die Frage ,Wer bin ich?“.

Angesichts dessen widmet sich das folgende Forum dem Thema „Multiple Identitäten in modernen Gesellschaften“. Jürgen Straub nähert sich aus der theoretischen Perspektive der Begrifflichkeit und unterschiedlichen Konzepten von Identität(en) und zeigt auf, wie sich angesichts der gegenwärtigen, mannigfaltigen Herausforderungen an das Individuum und die Gesellschaft Identität heute verstehen lässt. Aus der politischen Systemperspektive nimmt Sabine Riedel beispielhafte Konflikte um nationale Identitäten in der EU in den Blick und plädiert angesichts des Zulaufs für nationalistische und separatistische Bewegungen für eine Stärkung der Selbstverantwortung der Nationalstaaten und deren Nationen. Armin Nassehi stellt schließlich aus der europäischen Perspektive die Frage, warum es trotz aller Bemühungen (noch) nicht zur Ausbildung einer tatsächlichen europäischen, transnationalen Identität gekommen ist. 